Conclusions For STI prevention programs to have relevance to younger boys, they need to tap into developmental changes in the cultural, relational, and behavioural contexts of sex.

\section{2-S1.02 IS CONCURRENCY THE NEW SERIAL MONOGAMY? EVIDENCE FROM A LARGE SURVEY OF PEOPLE ATTENDING CONTRASTING GENITOURINARY MEDICINE (GUM) CLINICS IN ENGLAND}

doi:10.1136/sextrans-2011-050109.68

${ }^{1} \mathrm{C}$ H Mercer, ${ }^{1} \mathrm{C}$ R H Aicken, ${ }^{2} \mathrm{~N}$ Low, ${ }^{3} \mathrm{C}$ S Estcourt, ${ }^{4} \mathrm{P}$ J White, ${ }^{5} \mathrm{~F}$ Keane, ${ }^{6} \mathrm{G}$ Brook, ${ }^{1} \mathrm{G}$ Rait, ${ }^{7} \mathrm{~J}$ A Cassell. ${ }^{1}$ University College London, London, UK; ${ }^{2}$ University of Bern, Bern, Switzerland; ${ }^{3}$ Barts and the London School of Medicine and Dentistry, London, UK; ${ }^{4}$ Health Protection Agency, London, UK; ${ }^{5}$ Royal Cornwall Hospitals NHS Trust, Truro, Cornwall, UK; ${ }^{6}$ Central Middlesex Hospital, London, UK; ${ }^{7}$ Brighton and Sussex Medical School, Brighton, UK

Background Concurrency is an important risk behaviour for STI transmission at the population level, yet few surveys ask detailed questions about concurrency. Even if partnerships do not overlap, there is potential for STI transmission if the gap between the end of one partnership and the start of the next is short. We examined the prevalence of concurrency, quantified the gap between partners and examined gender differences in a high-risk population.

Methods Cross-sectional survey of 2203 people attending four contrasting GUM clinics in England in 2009. Attendees completed a questionnaire including questions about their three most recent partnerships in the 3 months prior to attending GUM. Gaps between partners were calculated as the time between most recent sex with a previous partner and first sex with a more recent partner. Partnerships were considered concurrent if the gap was negative.

Results $92 \%$ of attendees reported sex partner(s) in the last 3 months, yet, while the median number of partners was 1387 men $(48 \%)$ and 294 women $(28 \%)$ reported $2+$ partners in this time $(p<0.001)$. For $49 \%$ of these attendees, $\geq 2$ of their 3 most recent partners were concurrent ( $p=0.07$ for gender difference). Among the 347 concurrent partnerships reported, the median overlap was long: 113 days (IOR: 28-460 days) with just 40 partnerships (12\%) involving an overlap of $<2$ weeks (no significant gender difference). $40 \%$ of attendees with concurrent partners had not used condoms consistently with either partner, and a further $24 \%$ of attendees with concurrent partners reported not using condoms at all with either partner. In addition (see Abstract O2-S1.02 figure 1), while $78 \%$ of men's partners and $70 \%$ of women's were overlapping or had a brief gap ( $<2$ weeks), just $12 \%$ and $20 \%$ respectively involved a gap between partners of $>4$ weeks.

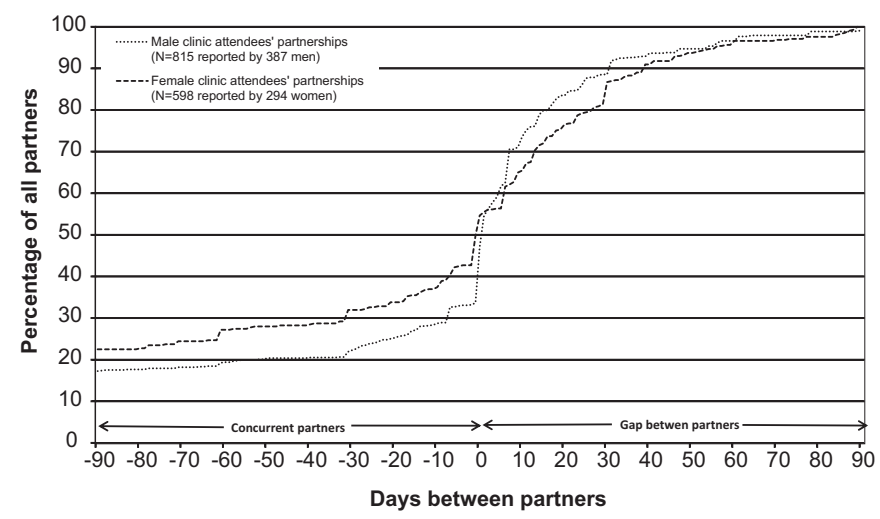

Abstract 02-S1.02 Figure 1 Cumulative percentage distribution of the time between partners in the 3 months prior to attending GUM clinic reported by attendees with $2+$ partners, by gender.
Conclusions The majority of GUM attendees reported few partners in the last 3 months, yet half of those with $2+$ partners had concurrent partnerships, such that concurrency is as common as serial monogamy in this population. These partnerships typically overlapped by a number of weeks and condoms were seldom used, if at all. Among the serially monogamous, sex with a new partner within days of last sex with a former partner was common so the potential for STI transmission is high. Understanding concurrency and the gaps experienced by high-risk individuals is important for ensuring that health promotion and partner notification are delivered appropriately and effectively.

\section{2-S1.03 ABC FOR PEOPLE WITH HIV: A LONGITUDINAL QUALITATIVE STUDY OF RESPONSES TO SEXUAL BEHAVIOUR RECOMMENDATIONS AMONG PEOPLE RECEIVING ANTIRETROVIRAL THERAPY IN JINJA, UGANDA}

doi:10.1136/sextrans-2011-050109.69

${ }^{1} \mathrm{C}$ Allen, ${ }^{2} \mathrm{M}$ Mbonye, ${ }^{3} \mathrm{~J}$ Seeley, ${ }^{4} \mathrm{~J}$ Birungi, ${ }^{5} \mathrm{~B}$ Wolff, ${ }^{6} \mathrm{~A}$ Coutinho, ${ }^{7} \mathrm{~S}$ Jaffar. ${ }^{1}$ Caribbean HIVqAIDS Alliance St Michael, Barbados; ${ }^{2}$ Medical Research Council /Uganda Virus Research Institute, Entebbe, Uganda; ${ }^{3}$ University of East Anglia, Kampala, UK; ${ }^{4}$ The AIDS Support Organisation, Kampala, Uganda; ${ }^{5}$ Medical Research Councill Uganda Virus Research Institute, Entebbe, Uganda; ${ }^{6}$ Infectious Diseases Institute, Kampala, Uganda; ${ }^{7}$ London School of Hygiene and Tropical Medicine, London, UK

Background People living with HIV (PLHIV) taking ART are increasingly involved in positive prevention" initiatives. These are generally oriented to promoting Abstinence, "Being faithful" (partner reduction) and condom use.

Methods We conducted a longitudinal qualitative study with PLHIV using ART, who were provided with adherence education and counselling support by a Ugandan non-governmental organisation, The AIDS Service Organisation (TASO). Participants were 40 people selected sequentially as they started ART, stratified by sex, ART delivery mode (clinic- or home-based), and HIV progression stage (early or advanced). At enrolment and 3, 6, 18 and 30 months, semistructured interviews and home observation visits explored adherence and life changes.

Results At initiation of ART, participants agreed to follow TASO's positive-living (similar to $A B C$ ) recommendations. Initially poor health prevented sexual activity. As health improved, participants prioritised resuming economic production and support for their children. With further improvements, sexual desire resurfaced and people in relationships cemented these via sex. Married male participants were able to use condoms with their wives, but married female participants were unable to assure consistent condom use despite serostatus disclosure to their husbands. Several participants sought and some obtained HIV positive partners (serosorting) for emotional security, support in ART adherence, sexual fulfilment, avoidance of stigma and sometimes parenthood. Respondents used condoms in the early period of new relationships, but several did not disclose HIV status to partners at first. Male partners of female respondents stopped using condoms after a variable initial period. This contributed to continued non-disclosure, since the women feared violence or loss of economic security from disclosure.

Conclusion Positive prevention interventions continue to concentrate on behavioural outcomes and education and counselling approaches. Our findings show that, as ART leads to health improvements, gender norms, economic needs, sexual desires and a wish for "normalisation" of social roles and relationships increasingly influence sexual behaviour. Positive Health, Dignity and Prevention require combinations of appropriate biological, behavioural and structural interventions tailored to the economic and cultural milieu and informed by an appreciation of the human rights of PLHIV. 